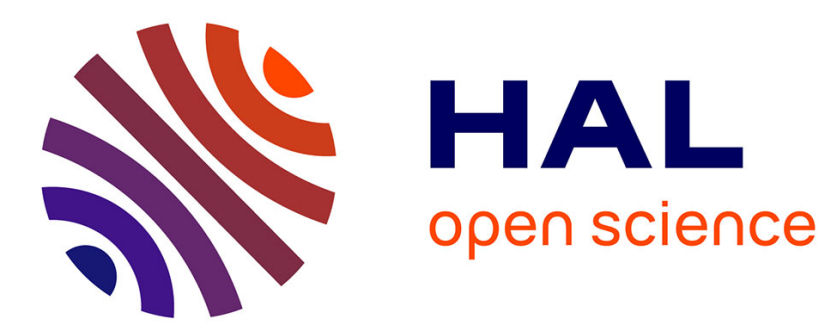

\title{
Ultrasonic seismic imaging of lava samples by viscoacoustic asymptotic waveform inversion: calibration and developments
}

G Saracco, Alessandra Ribodetti, Solène Turquety, Frédéric Conil

\section{- To cite this version:}

G Saracco, Alessandra Ribodetti, Solène Turquety, Frédéric Conil. Ultrasonic seismic imaging of lava samples by viscoacoustic asymptotic waveform inversion: calibration and developments. 17th IEEE Instrumentation and Measurement Technology Conference, May 2000, Baltimore, United States. pp.380-385, 10.1109/IMTC.2000.846890 . hal-01766760

\section{HAL Id: hal-01766760 \\ https://hal.science/hal-01766760}

Submitted on 18 Apr 2018

HAL is a multi-disciplinary open access archive for the deposit and dissemination of scientific research documents, whether they are published or not. The documents may come from teaching and research institutions in France or abroad, or from public or private research centers.
L'archive ouverte pluridisciplinaire HAL, est destinée au dépôt et à la diffusion de documents scientifiques de niveau recherche, publiés ou non, émanant des établissements d'enseignement et de recherche français ou étrangers, des laboratoires publics ou privés. 


\title{
Ultrasonic Seismic Imaging of lava samples by Viscoacoustic Asymptotic Waveform Inversion: Calibration and Developments
}

\author{
Ginette SARACCO, \\ CNRS-UPR 4661, Geophysique Interne, Campus de Beaulieu, bat 15, F-35042 Rennes cedex, France. Phone: \\ +33299286379, e-mail: ginet@univ-rennes1.fr \\ Alessandra RIBODETTI \\ CNRS-UMR 6536, Geosciences Azur, Universite de Nice-Sophia Antipolis, BP 48, 2 quai de la darse, F06235 \\ Villefranche/mer cedex France. Phone: + 33493763882 ,e-mail: ribodeti@obs-vlfr.fr \\ Solene TURQUETY \\ CNRS-UPR 4661, Geophysique Interne, Campus de Beaulieu, bat 15, F-35042 Rennes cedex, France Phone: \\ +3329928 2637, email: turquety@univ-rennes1.fr

\section{Frederic CONIL} \\ CNRS-UPR 4661, Equipe de Geophysique Interne, Campus de Beaulieu, bat 15, F-35042 Rennes cedex, France \\ Phone: + 33299281457 , email: fconil@univ-rennes1.fr
}

\begin{abstract}
The object of this study is to estimate the viscoelastic parameters (compressional velocity and the intrinsic attenuation factor $Q$ ) of lava samples in acoustic tomography experiments by the use of an asymptotic viscoacoustic diffraction tomography method developed by Ribodetti et al 2000. 2.5-D common-offset ultrasonic experiments are performed in a water tank laboratory.
\end{abstract}

Keywords - intrinsic attenuation, asymptotic and Born approximation, viscoacoustic medium, diffraction tomography method.

\section{INTRODUCTION}

In laboratory, the methods generally used for measuring attenuation can be separated into the following categories: free vibrations, forced vibrations, observation of stressstrain curves and wave propagation methods (see [3], [7], [11] for a review). The method presented in this paper belongs to the category of wave propagation method.

The use of this method in the lower ultrasonic frequency range is of particular interest since the loss parameters involved closely parallel to those measured in field experiments.

In general, wave propagation experiments can be classified according to whether they make use of pulse-echo or through-transmission methods. These methods assume the amplitude of seismic wave (generally considered to be a plane wave) decays exponentially with distance or time and that one can correct for losses other than intrinsic attenuation. The extraneous losses include beam spreading, coupling losses, diffraction losses, and wedging losses. For the pulse-echo technique, the attenuation is found by measuring the amplitude decay of multiple reflections from a free surface. The advantage of this method is the use of the absolute amplitude which allows to analyze easily the behavior of the pulse decay. This method has been used successfully for determining $Q$ value of single crystals and to study some fine-grained limestones (Peselnick and Zietz, 1959).

In transmission experiments, the transducers are located at the opposite ends of two samples of different thickness. The attenuation obtained represents an average along the variation of the thikness. In many cases, diffraction losses may become important. Because of beam spreading, internal reflections and mode conversion may occur, interfering with the direct wave. Diffraction may cause problems even with pulse transmission techniques. These techniques are fraught with experimental and interpretational difficulties. The viscoacoustic tomography provided both a velocity and $Q$ images of the target. We verified the efficiency of the inversion by comparing the observed and the computed data after one iteration. An excellent match between the two data sets was obtained after several iterations.

This paper is organized as follows. In the part $\mathrm{II}$, we 
the computed data after one iteration. An excellent match between the two data sets was obtained after several iterations.

This paper is organized as follows. In the part II, we present the experimental setup to image the rocks samples. In the third part, we recall the method used to extract the rheological parameters. The results on lava samplesafter one iteration (andesite of Volvic see [1]) are presented in the last part.

\section{CALIBRATION AND EXPERIMENTAL DEVELOPMENT}

Experiments are performed in a water tank (dimensions 2.x1.6x1.5m) Laboratory at Rennes. The cylindrical lava sample to be imaged $(10 \mathrm{~cm}$ of diameter and $50 \mathrm{~cm}$ of lenght) is immersed in the center of the tank. In order to image the whole samples lava, the source/receiver system should rotate around the object that is what has been done in previous experiments. For reasons of convenience and of accuracy, it is here the object that rotates and not the transducers, which is obviously equivalent if the axis of rotation is the center of the cylindrical object.

To avoid the multiple parasite reflections on the walls of the tank, we performed experiments between $342 \mathrm{kHz}<$ $f c<668 \mathrm{kHz}$ and $518 \mathrm{kHz}<f c<1.01 \mathrm{MHz}$. This needs to have a good precision in translation $10^{-2} \mathrm{~mm}$ and in rotation $<1^{\circ}$. Diffraction and transmission experiments are first carried out on homogeneous PVC samples in order to obtained a calibration of the emission-reception system (referency imaging of the source, spectral emission, sampling frequency), and geometrical configuration (rotation and shifting precision, offset source-receivers, sampling acquisition), see Fig.1, Fig. 3.

The wavelenght of investigation is between $4,33 \mathrm{~mm}$ and $0,148 \mathrm{~mm}$ in water, with a water velocity of $1482.89 \mathrm{~m} / \mathrm{s}$ [4], and attenuation coefficient $\alpha=23.10^{-3} \mathrm{Neper} / \mathrm{m}$. The source signal generated by the synthetizer is a Ricker function in time (seconde derivative of a Gaussian function), for two different central frequencies $f c=500 \mathrm{kHz}$ and $750 \mathrm{kHz}$ before to be amplified and send to the directional hydrophone-source. The attenuation factor $Q$ is then respectively around 46055.7 and 69083.5.

The object to be imaged is constituted by a cylindrical lava sample set vertically in the water tank Fig. 2.

The hydrophone-source emits ultrasonic waves towards the object, and the scattered pressure field is then measured by a second transducer. 72 seismograms per common-offset gather are recorded see Fig.4, with an angular step of 5 degrees. For each step we average over 16 recorded signals and one complete acquisition $\left(0<\theta<360^{\circ}\right)$ takes around $30 \mathrm{mn}$. These response are digitazed with a sampling rate of $2.10^{-8} s$ and recorded by the A/D converter (50000 samples per trace), before to be stored on the computer. The transducers have an azimuthal offset of $20^{\circ}$ and are located from the lava sample

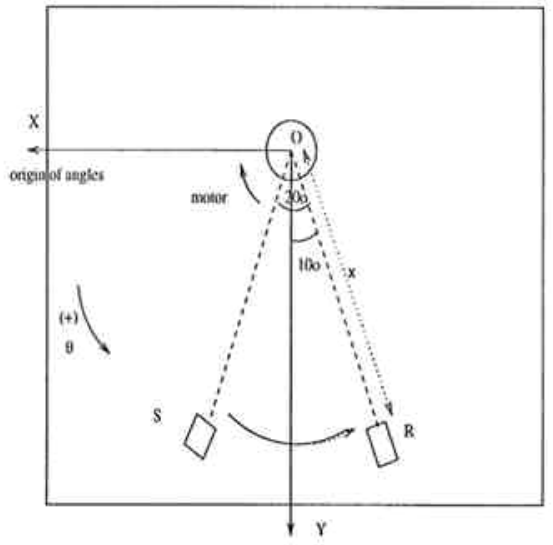

Fig. 1. Geometric configuration for the system source-receiver

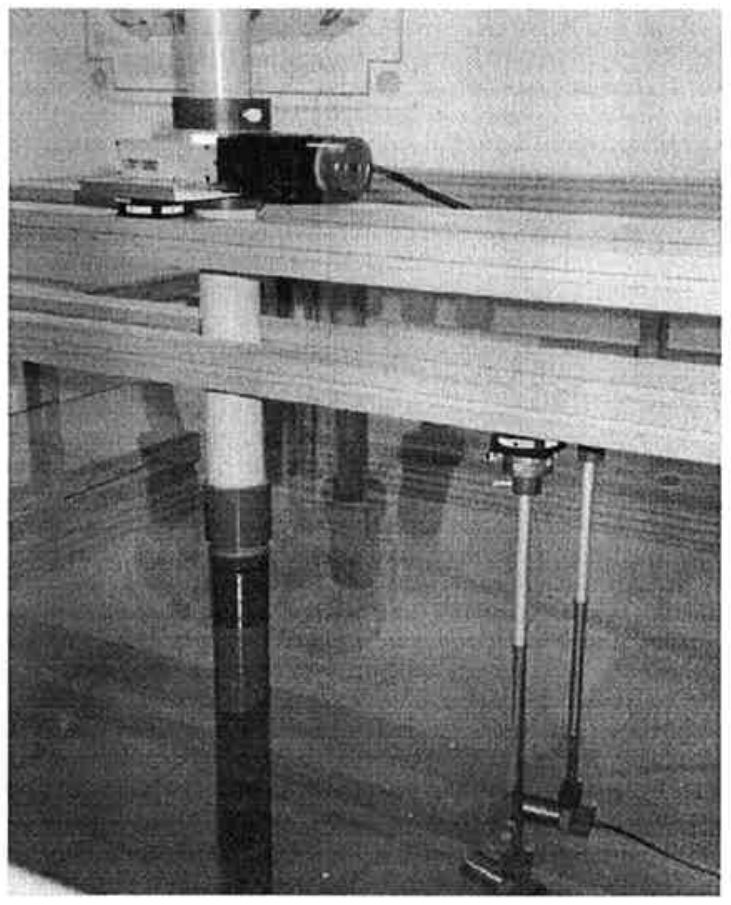

Fig. 2. Water tank experiment, computer-based control and data acquisition systems

center at $34 \mathrm{~cm}$. The whole sample is then insoned by the wave front issued from the directional source. Beforehand, the source signal is measured and recorded in the same conditions (sample frequency $(50 \mathrm{MHz})$, distance sourcereceiver, temperature of water $20.2^{\circ} \mathrm{C}$, ambiant noise) to performe a deconvolution of data experiments.

\section{NUMERICAL METHOD.}

The technique for recovering the velocity $\mathrm{c}$ and the attenuation factor $\mathrm{Q}$ of rocks samples is based on the high frequency asymptotic ray theory combined with a linearized method using a gradient method (see [5], [6], [8]). The ray theory allows the fast computation of the Green's function between the source and the scatterer as well as between 


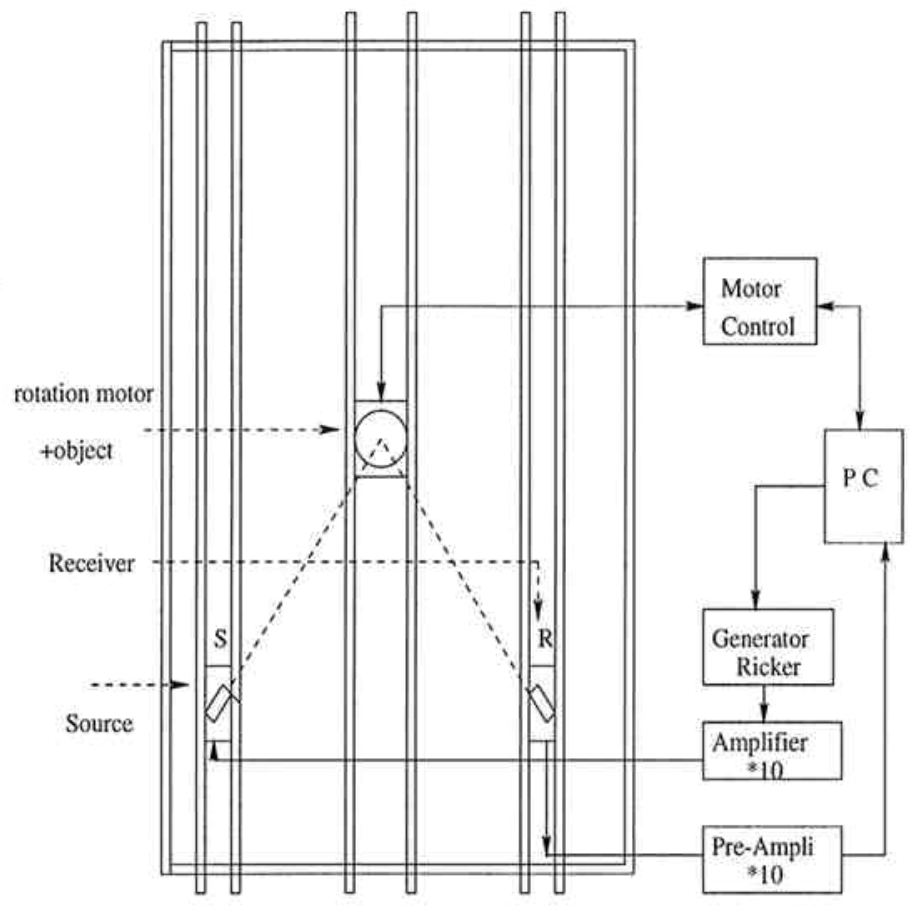

Fig. 3. Schema of the water tank: Geometric configuration and data acquisition system

the scatterer and the receiver, while the Born approximation allows us to compute the medium parameters as the compressional velocity and the intrinsic attenuation. In order to recover in few iterations these quantities an accurate estimation of the Hessian developed by Ribodetti is used. The reader will find a complete developement in [9] [10]. The acoustic case is related in the work of [12], [13]. We just recall the principal step to recover the rheological perturbations.

Let $P(x, \omega)$ the acoustic pressure measured at the point $x$ for the temporal frequency $\omega$. We have a linear relation between the Green's function $\mathcal{G}$ solution of the wave equation and the source term $\mathcal{F}$ :

$$
P(\mathbf{x}, \omega)=\int \mathcal{G}(\mathbf{s}, \mathbf{x}, \omega) \mathcal{F}(\mathbf{s}, \omega) d \mathbf{s}
$$

Introducing $\tilde{c}$ the complex velocity ([2], [11]), we can taking into account both the perturbations for $c$ and $Q$ :

$$
\frac{1}{\tilde{c}}=\frac{1}{c}\left(1+\frac{i}{2 Q} \operatorname{sign}(\omega)\right) .
$$

For small perturbations of the background medium compared with the wavelength of the source signal, we can write all quantities as $X=X_{o}+\delta X$.

The solution obtained from the Born approximation (ie $\left.P(\mathbf{x}, t)=P_{o}(\mathbf{x}, t)+o\left(\tilde{c}^{2}\right)\right)$, for the linearized forward problem in a viscoacoustic medium and frequency domain is:

$$
\delta P(\mathbf{s}, \mathbf{r}, \omega)=\omega^{2} \int_{\mathcal{M}} \mathcal{G}_{o}(s, x, \omega) \mathcal{G}_{o}(x, r, \omega)
$$

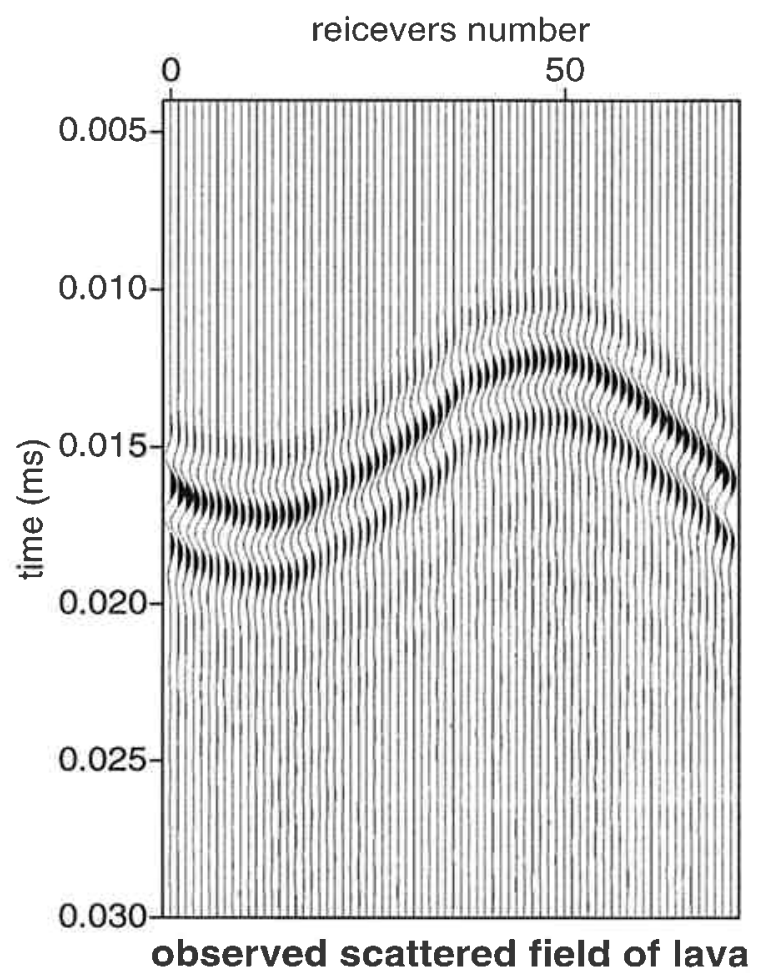

Fig. 4. Experimental scattered pressure field measured during 72 acquisitions with a central frequency for the source of $500 \mathrm{kHz}$

$$
\mathbf{K}(\mathbf{x}, \omega)\left(\begin{array}{c}
\delta c(\mathbf{x}) \\
\delta Q(\mathbf{x})
\end{array}\right) d \mathbf{x}
$$

where the integration domain is $\mathcal{M}$ and $\mathbf{K}(x, \omega)$ represents the vector components which depends only on the rheology of the background medium. It is obtained after a first order limited development of (2). The Green's function $\mathcal{G}_{0}$ taken under asymptotic ray theory, is computed analytically in the homogeneous background medium (water). We can write the perturbated pressure in this form:

$$
\begin{gathered}
\delta P(\mathbf{s}, \mathbf{r}, \omega)=\mathcal{K}(\omega) \int_{\mathcal{M}} \mathcal{A}(\mathbf{r}, \mathbf{x}, \mathbf{s}) e^{i \omega \mathcal{T}(\mathbf{r}, \mathbf{x}, \mathbf{s})} \\
e^{-\alpha(\mathbf{r}, \mathbf{x}, \mathbf{s})|\omega|} \mathbf{K}(\mathbf{x}, \omega)\left(\begin{array}{c}
\delta c(\mathbf{x}) \\
\delta Q(\mathbf{x})
\end{array}\right) d \mathbf{x} .
\end{gathered}
$$

where $\mathcal{A}, \mathcal{T}$ and $\alpha$ represent respectively the geometrical amplitude, the travel time and the total attenuation associated to the ray under asymptotic condition. The perturbation is then

$$
\delta P=\mathcal{G} \mathbf{f}_{\mathcal{M}} \quad \text { where } \quad \mathbf{f}_{\mathcal{M}}(\mathbf{x})=\left(\begin{array}{c}
\delta c(\mathbf{x}) \\
\delta Q(\mathbf{x})
\end{array}\right) .
$$

Following the approach of [5], the velocity and the attenuation perturbations are obtained by iterative least squares minimization of the weighted misfit between the measured and computed pressure using a quasi Newtonian algorithm, and it is given by: 


$$
\mathbf{f}_{\mathcal{M}}=\left[\mathcal{G}^{*} \mathcal{Q G}\right]^{-1} \mathcal{Q} \mathcal{G}^{*}\left(\delta P_{\text {meas }}-\delta P_{\text {cal }}\right)
$$

The iterative inversion is linear since the background medium is constant over iterations. $\mathrm{Q}$ is the weighting cost function. After diagonalization of the Hessian (see Ribodetti et al 1999), the perturbations $\mathbf{f}(x)$ are obtained by a weighted diffraction average of the data misfit:

$$
\Delta \delta P(\phi, \omega)=\delta P_{\text {meas }}(\phi, \omega)-\delta P_{\text {cal }}(\phi, \omega)
$$

Let the discretized expression calculated over positive frequencies

$$
\begin{aligned}
& \mathbf{f}(\mathbf{x})=-\mathbf{R}^{-1} \sum_{\theta} \frac{\Delta \theta}{\mathcal{A}(\phi, \mathbf{x})} \sum_{\omega+} \Delta \omega\left|\frac{\partial \mathbf{k}}{\partial\left(\theta, \omega^{+}\right)}\right| \\
& e^{\left|\omega^{+}\right| \alpha(\theta, \mathbf{x})} \\
& \mathcal{R} e\left[i e^{-i \frac{p i}{4}} \omega \mathbf{K}^{\dagger}\left(\mathbf{x}, \omega^{+}\right) e^{-i \omega^{+}} \mathcal{T}(\theta, \mathbf{x}) \Delta \delta P\left(\theta, \omega^{+}\right)\right]
\end{aligned}
$$

where $\mathcal{R} e$ denotes the real part of a complex number, $\theta$ the angular step, $\mathbf{k}$ the wave number and $\mathbf{R}$ is Hessian matrix obtained (see [12], [10] ) after diagonalization and conditionnement to 1 .

Since the Hessian matrix is

$$
\mathbf{R}=\mathbf{K}^{\dagger} \mathbf{K}=\mathbf{K}_{-}^{\dagger} \mathbf{K}_{-}+\mathbf{K}_{+}{ }^{\dagger} \mathbf{K}_{+}
$$

with

$$
\mathbf{K}(\mathbf{x}, \omega)=(a+i \operatorname{sgn}(\omega) b \quad c+i \operatorname{sgn}(\omega) d)
$$

and

$$
\begin{array}{cc}
a=-\frac{2}{c_{o}^{3}}\left(1-\frac{1}{4 Q_{o}^{2}}\right) & b=-\frac{2}{c_{o}^{3} Q_{o}} \\
c=\frac{1}{2 c_{o}^{2} Q_{o}^{3}} & d=-\frac{1}{c_{o}^{2} Q_{o}^{2}}
\end{array}
$$

We can write $\mathbf{R}$ under the form:

$$
\mathbf{R}=2\left(\begin{array}{cc}
A=a^{2}+b^{2} & B=a c+b d \\
B=a c+b d & C=c^{2}+d^{2}
\end{array}\right)
$$

Let $\mathbf{R}_{\mathbf{o}}$ the matrix previously defined and $\mathbf{R}$ the well conditionned matrix then

$$
\mathbf{R}=\mathbf{N R}_{\mathbf{o}} \mathbf{N}^{t} \text { where } \mathbf{N}=\left(\begin{array}{cc}
e & f \\
0 & g
\end{array}\right)
$$

and

$$
e=\sqrt{|A|}, f=\frac{B}{\sqrt{|A|}}, \quad g=\sqrt{\frac{\left|\operatorname{det} \mathbf{R}_{\mathrm{o}}\right|}{|A|}} .
$$

Remark: $g$ can be very small but it is essential for the conditionnement of the matrix, so it must be defined accuratly.

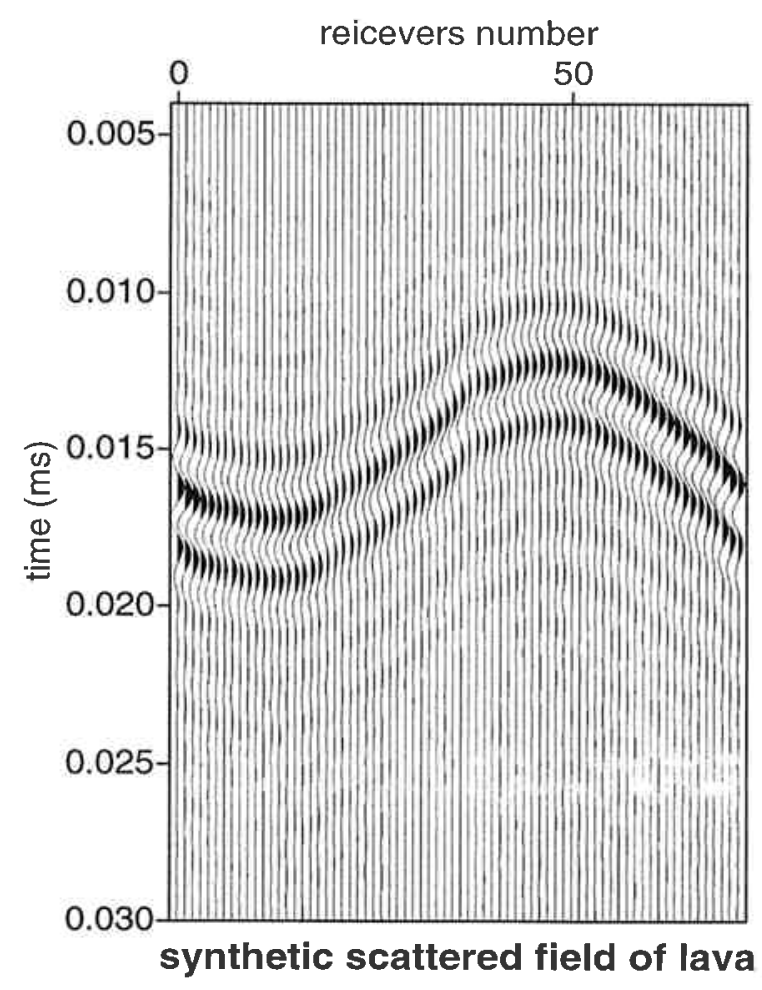

Fig. 5. Computed scattered field of the lava sample during 72 acquisitions after 1 iteration, $\mathrm{fc}=500 \mathrm{kHz}$.

\section{RESULTS}

Some previous transmission experiments allowed us to find a first and average estimation of the velocity and the attenuation of the object to be imaged. From 2.5D diffraction experiments of Lava samples from Volvic performed around two central frequencies fc of $500 \mathrm{kHz}$ and $750 \mathrm{kHz}$, and frequency range of $\mathrm{fc} / 2$, we have obtained very good images of the scattered pressure field without multiple reflections of the tank and good ratio signal over noise. After inversion and deconvolution of those experimental data using the ray Born approximation method we find in comparison with synthetic images obtained see Fig 5 , a correct localisation of wave front of the object in each direction (seismic trace). The map of perturbations obtained for lava samples of $10 \mathrm{~cm}$ of diameter are shown Fig 6 and Fig 7 respectively for the compressional velocity and the attenuation factor. The deconvolution reduces the range of velocity and attenuation by a factor of $10^{-3}$ (Cf.8 and 9. In conclusion the compressional velocity and the attenuation factor are obtained respectively with a correct estimation for the one, but a more important deviation in the estimation for the second one presenting some oscillations.

The heterogeneous nature of the sample shows that for a complete study a post processing method integrating a frequency dependance of the object is perhaps necessary but this previous results on real data are encouraging. 


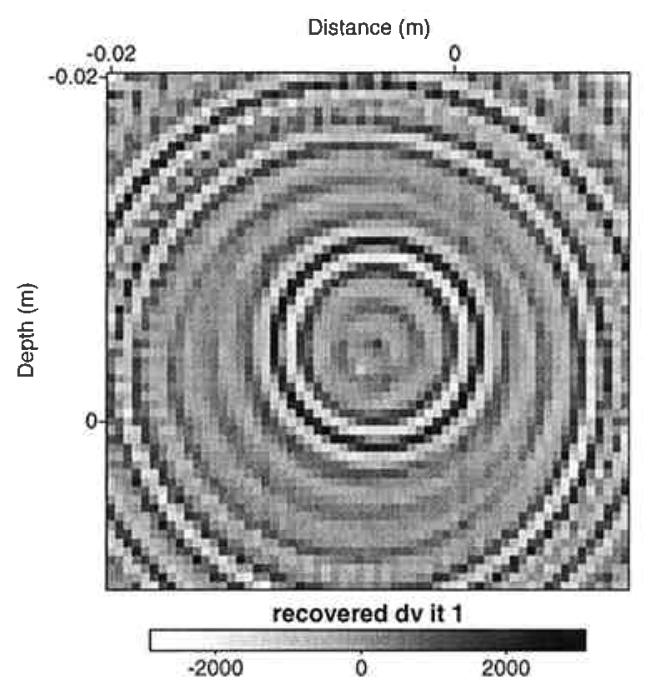

Fig. 6. Map of compressional velocity perturbations on Lava sample of volvic: Results after one iteration

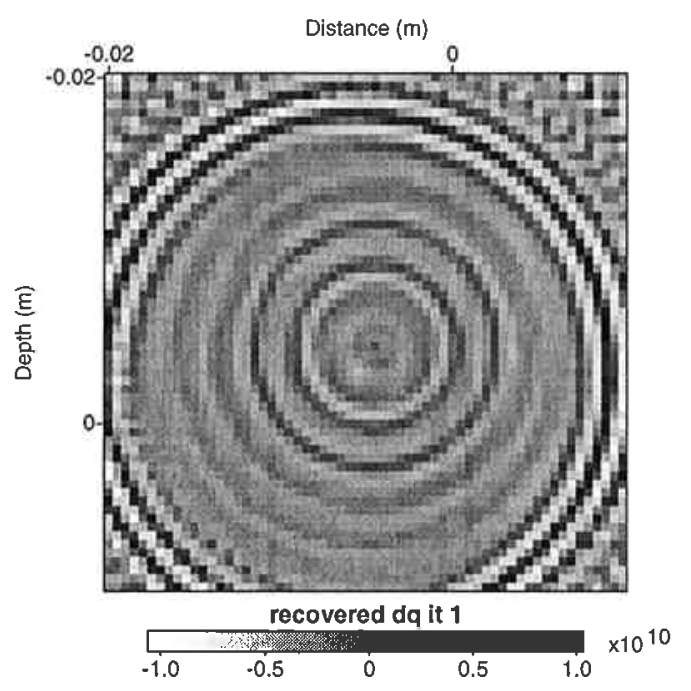

Fig. 7. Map of attenuation factor perturbations obtained on lava sample of volvic: Results after one iteration

\section{CONCLUSION}

The experimental setup and the processing developed in this study could be used as a tool for rock properties estimation. The wave-propagation method (see referencies below) may present the following advantages for rock properties analysis: -First, compared to wave-propagation methods using transmitted waves, the results obtained with diffraction tomography may be extrapolated more easily to the scale of seismic reflection experiments because of the analogy of the propagation mode (namely, reflection mode) used in the two kinds of experiments. -Second, the experimental setup is particularly adaptable, and reproducible. We have the choice of the geometric configuration (transmitted or reflected), the offset

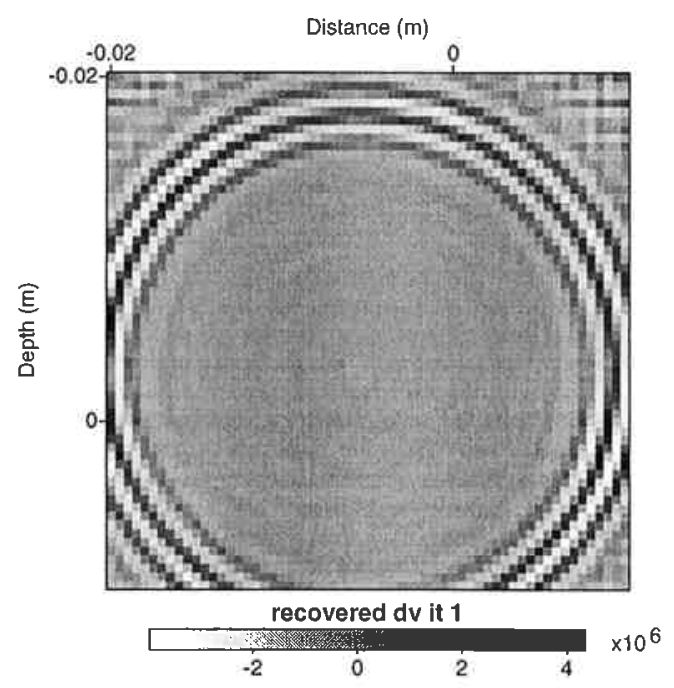

Fig. 8. Map of compressional velocity perturbations on lava sample of volvic

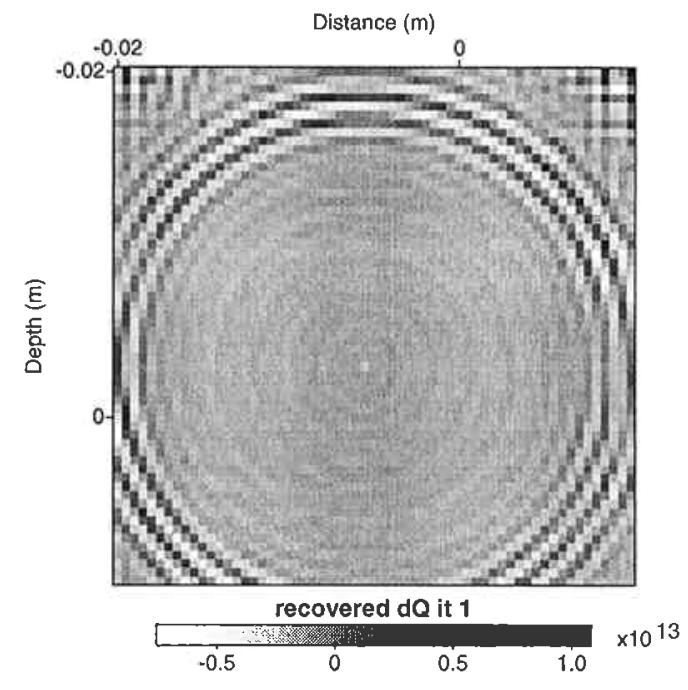

Fig. 9. Map of attenuation perturbations on lava sample of Volvic: Results after one iteration without deconvolution

(source-receiver) and we have the controle of the acoustic source signal send to the hydrophone. The background medium is composed of water and then it is easy to control its properties, such as temperature, to control the source signal and the ratio source over noise. It is also easy to separate the wavefield scattered by the target from the other signals by performing a dual acquisition (namely, with and without the target in the water tank). The Ray-Born approximation combined with an accurate estimation of the Hessian allows us to obtain after few iteration (1 or 2) a good accordance in amplitude and location between the calculated field and the observed field. For a complete quantitative study of lava sample (heterogeneous nature), it seems necessary to include a post processing method integrating a frequency dependance of the object but this previous results on real data are en- 
couraging.

\section{ACKNOWLEDGMENTS}

This work was principally supported by European Commission, on European project TomoVes ENV4-98/00698 and we would like to thank M. Zamora from IPGP for the samples of andesite she lended us for our experiments, and J.Virieux from Sophia-Antipolis University for his numerous and constructive discussions.

\section{References}

[1] Bernard, M.-L., PhD in applied geophysics, 1999 at Inst. Phys. du Globe-Paris, Experimental study of physical properties of Pyroclastiques rocks of Pelee Mountain.

[2] Chang, H., McMechan, G. Numerical simulation of multiparameter seismic scattering: Bull. Seis. Soc. Am., 86, 18201829,1996

[3] Fujii, K., Masui, R. Accurate mesurements of the sound velocity in pure water by combining a coherent phase-detection technique and a variable path-length interferometer: J. Acoust. Soc. Am., 93, 273-282, 1993

[4] Greenspan, M., Techiego, C. E. Tables of speed of sound in water (J. Research. Nat. Bur. Standards), 59, pg. 75, 1957

[5] Jin, S., Madariaga, R., Virieux, J., Lambaré, G. Twodimensional asymptotic iterative elastic inversion: Geophys. J. Int., 108, 1-14, 1992

[6] Lambaré, G. Virieux, J., Jin, S., Madariaga, R. Iterative asymptotic inversion in the acoustic approximation: Geophysics, 57, 1138-1154, 1992

[7] Lo, T., Toksöz, M. N., Xu, S., Wu, R. S. Ultrasonic laboratory tests of geophysical tomographic reconstruction: Geophysics, 53, $947-956,1988$

[8] Ribodetti, A., Virieux, J., Durand, S. Asymptotic theory for viscoacoustic seismic imaging: 65th Ann. Internat. Mtg., 65th Ann. Internat. Mtg., Expanded Abstracts, Soc. Expl. Geophys., 631-634, 1995

[9] Ribodetti, A.,PhD in applied geophysics, 1998 at the Université de Nice-Sophia Antipolis (FR) "3D Asympotic Seismic Imaging".

[10] Ribodetti A., Operto S., Virieux J., Lambare G., Valero HP and Gibert D., Asymptotic viscoacoustic diffraction tomography of ultrasonic laboratory data: a tool for rock properties analysis, Journ. Geophys. Int., 140 (2), 324-340, feb. 2000

[11] Toksöz, M.N., Johnston, D.H. Seismic wave attenuation: Soc. Expl. Geophys., Tulsa, 1981

[12] Forgues, E., ,PhD in applied geophysics, 1996 at Inst. Phys. du Globe-Paris, Multiparameters Linearized Inversion via the ray Theory: Application to seismic data, Surface reflection.

[13] Thierry, P., ,PhD in applied geophysics, 1997 at Inst. Phys. du Globe-Paris, 3D Migration/Inversion in depth with preserved amplitude: Application to seismic data, reflection before stack. 